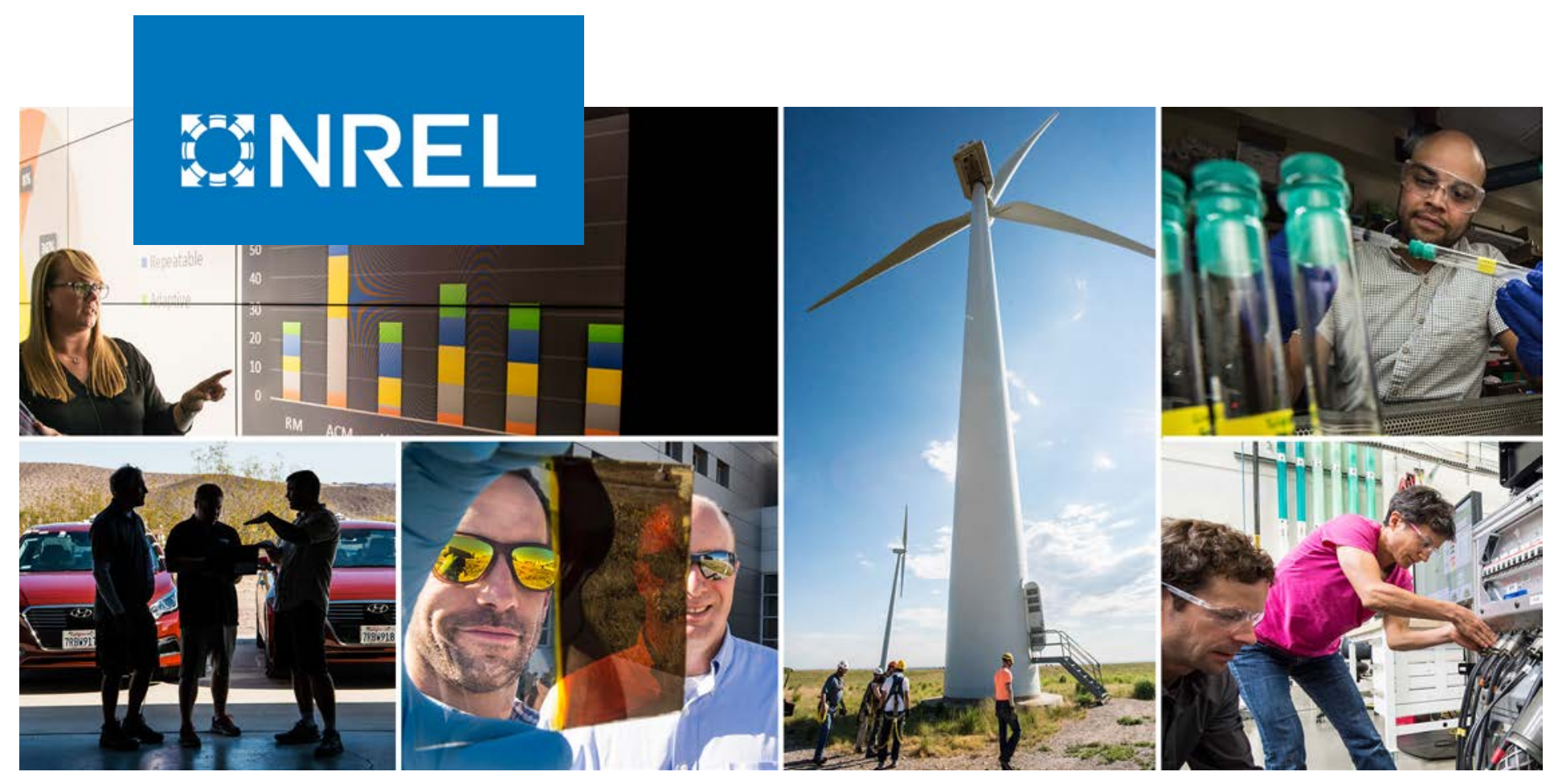

\title{
What Is the Value of Alternative Methods for Estimating Ramping Needs?
}

\section{Preprint}

Evangelia Spyrou, ${ }^{1}$ Venkat Krishnan, ${ }^{1}$ Qingyu Xu, ${ }^{2}$ and Benjamin F. Hobbs ${ }^{2}$

${ }^{1}$ National Renewable Energy Laboratory

2 Johns Hopkins University

Prepared for the the 2020 IEEE Green Technologies Conference (IEEE GreenTech)

NREL is a national laboratory of the U.S. Department of Energy

Office of Energy Efficiency \& Renewable Energy

Operated by the Alliance for Sustainable Energy, LLC

This report is available at no cost from the National Renewable Energy Laboratory (NREL) at www.nrel.gov/publications.

\section{Conference Paper}

NREL/CP-5D00-75544

August 2020 


\title{
BNREL
}

\section{What Is the Value of Alternative Methods for Estimating Ramping Needs?}

\section{Preprint}

\section{Evangelia Spyrou, ${ }^{1}$ Venkat Krishnan, ${ }^{1}$ Qingyu Xu, ${ }^{2}$ and Benjamin F. Hobbs ${ }^{2}$}

\author{
${ }^{1}$ National Renewable Energy Laboratory \\ 2 Johns Hopkins University
}

\section{Suggested Citation}

Spyrou, Evangelia, Venkat Krishnan, Qingyu Xu, and Benjamin F. Hobbs. 2020. What Is the Value of Alternative Methods for Estimating Ramping Needs?: Preprint. Golden, CO: National Renewable Energy Laboratory. NREL/CP-5D00-75544.

https://www.nrel.gov/docs/fy20osti/75544.pdf.

(C) 2020 IEEE. Personal use of this material is permitted. Permission from IEEE must be obtained for all other uses, in any current or future media, including reprinting/republishing this material for advertising or promotional purposes, creating new collective works, for resale or redistribution to servers or lists, or reuse of any copyrighted component of this work in other works.

NREL is a national laboratory of the U.S. Department of Energy Office of Energy Efficiency \& Renewable Energy Operated by the Alliance for Sustainable Energy, LLC

This report is available at no cost from the National Renewable Energy Laboratory (NREL) at www.nrel.gov/publications.

Contract No. DE-AC36-08GO28308
Conference Paper

NREL/CP-5D00-75544

August 2020

National Renewable Energy Laboratory 15013 Denver West Parkway Golden, CO 80401

303-275-3000 • www.nrel.gov 


\section{NOTICE}

This work was authored in part by the National Renewable Energy Laboratory, operated by Alliance for Sustainable Energy, LLC, for the U.S. Department of Energy (DOE) under Contract No. DE-AC36-08G028308.Funding provided by U.S. Department of Energy Office of Energy Efficiency and Renewable Energy Solar Energy Technologies Office. The views expressed herein do not necessarily represent the views of the DOE or the U.S. Government. The U.S. Government retains and the publisher, by accepting the article for publication, acknowledges that the U.S. Government retains a nonexclusive, paid-up, irrevocable, worldwide license to publish or reproduce the published form of this work, or allow others to do so, for U.S. Government purposes.

This report is available at no cost from the National Renewable Energy Laboratory (NREL) at www.nrel.gov/publications.

U.S. Department of Energy (DOE) reports produced after 1991 and a growing number of pre-1991 documents are available free via www.OSTI.gov.

Cover Photos by Dennis Schroeder: (clockwise, left to right) NREL 51934, NREL 45897, NREL 42160, NREL 45891, NREL 48097, NREL 46526.

NREL prints on paper that contains recycled content. 


\section{What Is the Value of Alternative Methods for Estimating Ramping Needs?}

\author{
Evangelia Spyrou, Venkat Krishnan \\ Power Systems Engineering Center \\ National Renewable Energy Laboratory, Golden, CO \\ elina.spyrou@nrel.gov
}

\author{
Qingyu Xu, Benjamin F. Hobbs \\ Department of Environmental Health and Engineering \\ The Johns Hopkins University \\ Baltimore, MD
}

This paper was accepted by the 2020 IEEE Green Technologies Conference (IEEE GreenTech) scheduled from April 1-3, 2020 in Oklahoma City. However, the conference was canceled due to COVID-19.

\begin{abstract}
Power system operators procure and deploy flexibility reserves or ramping products to address balancing needs caused by uncertainty and variability of load and generation. Existing methods estimate ramping needs using calendar information and historical forecast errors. Novel methods investigate if real-time weather information could inform ramping and other balancing requirements. This paper compares estimation methods for ramping requirements in theory and practice. The theoretical framework indicates when an alternative method could yield improved economic or reliability performance than existing methods by requiring lower or higher levels of ramping products. Preliminary simulations on a 118-bus test system for 4 days in May 2019 illustrate how system performance improves or deteriorates when ramping requirements are weather-informed (alternative) instead of calendar-based (baseline). Preliminary results suggest high variability in change of performance and underline the impact of additional factors, such as system conditions, on the realized performance change.
\end{abstract}

Index Terms-Flexibility, ramping product, renewable uncertainty, forecast errors, probabilistic forecasts, reliability.

\section{NOMENCLATURE}

\begin{tabular}{|c|c|}
\hline \multicolumn{2}{|l|}{ Sets } \\
\hline G & Generators, indexed by $g$ \\
\hline M & List of markets/scheduling algorithms \\
\hline RES & $\begin{array}{l}\text { List of reserve products, indexed by res. Includes } \\
\text { regulation up (RU), regulation down (RD), } \\
\text { spinning and nonspinning reserves }\end{array}$ \\
\hline$T$ & Time intervals, indexed by $t$ \\
\hline \multicolumn{2}{|l|}{ Parameters } \\
\hline$D_{M}$ & Duration of market interval of market $m$ in minutes \\
\hline$F R D R_{t}$ & Flexible ramp-down requirement at time $t$ \\
\hline$F R U R_{t}$ & Flexible ramp-up requirement at time $t$ \\
\hline$E R R O R_{\text {up } / \text { down }}$ & Net load forecast error used by baseline method \\
\hline HRTC & $\begin{array}{l}\text { Horizon of real-time unit commitment (here } 12 \\
\text { intervals) }\end{array}$ \\
\hline$L O A D_{t}$ & Electricity load at time $t$ \\
\hline$N L_{t, \text { measure }}$ & Statistical measure of net load at time $t$ \\
\hline$P M I N_{g}$ & Minimum generation level \\
\hline$P M A X_{g, t}$ & Maximum generation level \\
\hline$Q_{5 / 95}$ & $5^{\text {th }}$ and $95^{\text {th }}$ percentile of a random variable \\
\hline
\end{tabular}

\begin{tabular}{|c|c|}
\hline$R R_{g}$ & Ramping capability in MW/min \\
\hline$S O L A R_{t, \text { measure }}$ & Statistical measure of solar capability at time $t$ \\
\hline$W I N D_{t}$ & Forecast of total wind generation at time $t$ \\
\hline$W T P$ & Penalty price for deficits of flexible ramping product \\
\hline$\alpha$ & Averaging factor for 5-min ramping product: $\frac{\mathrm{D}_{R T U C}}{\mathrm{D}_{R T D}}$ \\
\hline$\beta$ & Averaging factor for $10-\mathrm{min}$ regulation product: $\frac{\mathrm{D}_{R T U C}}{10 \mathrm{~min}}$ \\
\hline \multicolumn{2}{|r|}{$10 \mathrm{~min}$} \\
\hline $\operatorname{costrd}_{t}$ & Penalty payments for the deficit in ramp-down \\
\hline $\operatorname{costru}_{t}$ & Penalty payments for the deficit in ramp-up \\
\hline$f r d_{g, t}$ & Ramp-down product provided by generator $g$ at time $t$ \\
\hline$f r d s_{t}$ & Violation of flexible ramp-down requirement at time $t$ \\
\hline$f r u_{g, t}$ & Ramp-up product provided by generator $g$ at time $t$ \\
\hline frus $_{t}$ & Violation of flexible ramp-up requirement at time $t$ \\
\hline$g e n_{g, t}$ & Dispatch level for generator $g$ at time $t$ \\
\hline$r s_{g, r e s, t}$ & Reserve of type res by generator $g$ at time $t$ \\
\hline$u_{g, t}$ & Binary variable for commitment of generator $g$ at time $t$ \\
\hline
\end{tabular}

\section{INTRODUCTION}

The changing energy mix, with increased contributions by uncertain and variable resources, has prompted changes in electricity market design and operator practices [1]. Power system operators assess solutions that help manage the uncertainty and variability of load and renewable generation. Among these are ramping products. Two independent system operators (ISOs)-Midcontinent [2] and California ISO (CAISO) [3] - have introduced a ramping product, and the Southwest Power Pool is currently developing one [4].

Past literature has demonstrated the ability of ramping products to manage uncertainty and variability. Reference [5] showed that ramping constraints can help operators meet expected variability; however, ramping constraints might not be cost-efficient when the deployment costs of ramping are not considered in the objective function that the market software optimizes. References [6] and [7] demonstrated how ramping constraints can assist system operators in managing uncertainty, but ramping constraints might be less cost-efficient than an "ideal" stochastic unit commitment method. Reference [8] discussed how the occurrence of variability and uncertainty can lead to energy imbalances and undesirable outcomes, such as power balance violations, real-time price spikes reflecting administrative penalties for violating constraints, leaning on regulation or interconnection, and out-of-market corrections. Moreover, [8] contended that a ramping constraint and product are more practical for managing uncertainty and variability than 
complex, time-consuming models and market formulations that fully capture stochastic processes and multiple possible futures.

However, the economic efficiency of ramping products depends on effective estimates of ramping requirements [6], [9]. In practice, ISOs use samples of past forecast errors and calendar information, such as hour and type of day, in order to estimate a parametric or empirical probability distribution function (PDF) of net load (load minus renewable generation) [3], [4]. Then, operators estimate either moments of parametric distributions [10] or percentiles [11] that they later use to determine ramping requirements. As systems change, ISOs assess the impact of additional factors on the PDF of net load. In particular, with the increase in variable energy resources (i.e., solar and wind generators), uncertainty related to forecasted generation by those resources might also increase. ${ }^{1}$ Weather conditions affect uncertainty related to forecasted generation by variable energy resources [12]. Therefore, state-of-the-art research is investigating whether real-time weather forecasts and measurements can be leveraged to estimate the net load PDF and associated balancing needs with increased accuracy.

For instance, CAISO is currently exploring the impact of weather variables on historical uncertainty of net load. Preliminary quantile regression analysis suggests that generation forecasts for variable energy resources and potentially temperature are statistically significant for the historical uncertainty of net load [13]. Reference [14] used forecasts by numerical weather prediction models to develop probabilistic forecasts of net load and concluded that weatherinformed probabilistic forecasts of net load can yield different (on average lower) balancing and regulation requirements than status-quo methods. Reference [15] reached a similar conclusion by integrating forecasts of renewable generation as well as weather parameters (irradiance, wind speed, temperature) into a dynamic sizing methodology for operating reserves in Belgium.

The effectiveness of such new sizing methods for balancing products ([14], [15]) has been assessed in two ways. First, researchers record how frequently the estimated requirements exceed the actual needs during a historical period, thereby estimating the reliability level of the proposed methods [14]. Second, researchers ([14], [15]) compare the estimated requirements to the status quo requirements. They hypothesize that higher (than the status quo) requirements will improve system reliability, whereas lower requirements will reduce costs; however, there are no reports in the literature of estimates of the value of alternative balancing product sizing methods in terms of quantitative reliability and economic performance. Therefore, the validity of the hypothesis is yet to be tested.

The contribution of this paper is in contrasting system performance under alternative methods for estimation of ramping needs (one type of balancing need) in theory and practice. In Section II, we develop a framework that summarizes all possible cases where differences in ramping requirements might explain differences in system performance. In Section III, we propose a metric that captures uncertaintyrelated system performance and is quantified via production cost simulations. In Section IV, we numerically illustrate the value of an alternative weather-informed method for the estimation of ramping requirements by comparing it to a baseline method on a case study with a 118-bus test system. Finally, in Section $\mathrm{V}$ we provide conclusions and areas for future research.

\section{The VAlue of Alternative RAMPING REQUiREMENT ESTIMATION METHODS}

Given the technical constraints of thermal and hydro generators with respect to startup, shutdown, minimum on/off time, and energy limits, day-ahead electricity markets or scheduling algorithms provide generator schedules usually with a lead time of approximately 14-38 hours; however, uncertainty and variability might lead to deviations from the day-ahead schedules. Balancing markets, such as real-time markets in CAISO [3] and intraday markets in Europe [16], as well as balancing products such as ramping products and regulation aim to address deviations and prevent energy imbalances by procuring flexibility and enabling the system to respond at lower cost to deviations from expected conditions. In this context, flexibility is defined as the load-following [17] ramping capability during a market interval starting from the last financially binding schedule. Therefore, a system needs flexibility or balancing capability equal to the system-wide net deviation of schedules. ${ }^{2}$

Sizing methods aim to estimate ramping requirements that will satisfy system flexibility needs within a target reliability level. Here, we compare system performance under an alternative set of ramping requirements (possibly provided by a weather-informed method) to the system performance under the baseline (status quo) method. The "alternative" method can estimate: (a) higher, (b) lower, or (c) identical requirements compared to the "baseline" method for each market interval.

Requirements under case (c) (identical) will not cause any differences in system performance between the two methods. Under cases (a) and (b), however, different levels of requirements between the two methods might lead to differences in system performance. We use "might" because differences in ramping requirements are necessary but not sufficient conditions for differences in system performance. In detail, the market software includes constraints that procure flexibility greater than or equal to the ramping requirements; hence, available flexibility could be higher than the requirements. Moreover, the market software allows for deficits, i.e., available flexibility lower than the requirements. In summary, the level of available flexibility, which depends on overall system conditions and the requirements, affects the system performance. For example, a system could be overly flexible during a market interval and its available flexibility could be higher than both sets of requirements, resulting in the same system performance under cases (a) and (b).

Fig. 1 summarizes all possible outcomes of the comparison of system performance under the alternative vs. the baseline estimation method and classifies market intervals in five categories: a1, a2, b1, b2, and c. In detail, if the realized

${ }^{1}$ A comprehensive list with all sources of uncertainty and variability is provided in [8].

\footnotetext{
${ }^{2}$ Depending on the congestion of the transmission system in question, the system might be divided into zones.
} 
ramping needs are less than the requirements of both methods (see case (2) of the framework shown in Fig. 1), both methods estimate adequate requirements for that market interval; hence, we do not anticipate any power balance violations caused by flexibility shortages and the method with the lowest requirements might incur lower production cost. If the realized ramping needs are more than the ramping requirements estimated by a method (see case (1) of the framework in Fig. 1 ), the system does not necessarily have enough resources ready to ramp up and system reliability might be at risk.

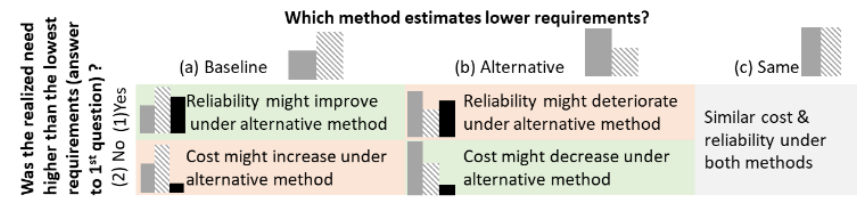

Figure 1. Framework classifying market intervals by comparing ramping requirements from two methods (baseline/alternative illustrated with solid/pattern gray bars) and the realized ramping needs (illustrated with solid black bars). Green, red, and gray boxes indicate potentially improved, deteriorated, and similar system performance, respectively, under the alternative ramping requirements.

\section{Quantifying The Value of Alternative Ramping REQUIREMENT ESTIMATION METHODS}

Analysts usually quantify system performance through production cost simulations, which minimize aggregate costs i.e., the sum of production cost (economic) and penalties for unserved energy (reliability). Analysts can formulate ramping products in tools such as the Flexible Energy Scheduling Tool for Integrating Variable Generation (FESTIV) [18] and conduct simulations with different sets of ramping requirements. FESTIV consists of a day-ahead scheduling algorithm run once per day and two rolling algorithms: realtime unit commitment (RTUC) and real-time dispatch (RTD). Following CAISO's conceptual design [11], we simulate a ramping product in FESTIV RTUC by adding (1)-(6) for $\forall \mathrm{t}$ $\in[1$, HRTC-1]:

$$
\begin{aligned}
& \text { costru }_{t} \geq W T P * \text { frus }_{t} \\
& \text { costrd }_{t} \geq W T P * \text { frds }_{t} \\
& \alpha * \sum_{g} \text { fru }_{g, t}+\text { frus }_{t} \geq F R U R_{t} \\
& \alpha * \sum_{g} f r d_{g, t}+f r d s_{t} \geq F R D R_{t} \\
& \text { gen }_{g, t}-\alpha * f r d_{g, t}-r s_{g, R D, t+1} \geq P M I N_{g} * u_{g, t+1} \\
& \text { gen }_{g, t}+\alpha * f r u_{g, t}+\sum_{r e s \neq R D} r s_{g, r e s, t+1} \leq P M A X_{g, t+1} * u_{g, t+1} \\
& \text { gen }_{g, t+1}-g e n_{g, t} \leq \alpha * f r u_{g, t} \\
& g e n_{g, t}-g e n_{g, t+1} \leq \alpha * f r d_{g, t} \\
& \alpha * f r u_{g, t}+\frac{\beta}{2} *\left(r s_{g, R U, t}+r s_{g, R U, t+1}\right) \leq R R_{g} * \mathrm{D}_{R T U C} \\
& \alpha * \operatorname{frd}_{g, t}+\frac{\beta}{2} *\left(r s_{g, R D, t}+r s_{g, R D, t+1}\right) \leq R R_{g} * \mathrm{D}_{R T U C}
\end{aligned}
$$

To concisely describe the formulation, we use (a) for upward and (b) for downward ramping. Eqs. 1 estimate the penalties $\left(\operatorname{costru}_{t}, \operatorname{costrd}_{t}\right)$ for ramping product deficits, which are added to the objective function of the production cost optimization. Eqs. (2) procure ramping, whereas eqs. (3) and (4) guarantee that the unit will not violate its minimum and maximum operating limits, respectively. Eqs. (5) count expected change in generation schedule toward the ramping product. Eqs. (6) ensure that the ramping capability for the units is shared among products. Slightly different constraints apply when the unit is starting up or shutting down. For example, when a unit shuts down, it can provide only frd equal to its downward scheduled movement and no $\mathrm{fru}$. Moreover, wind and solar are not eligible to provide ramping product, and hydro can provide ramping equal to its scheduled movement from $t$ to $t+1$. To ensure that flexibility produced in RTUC will be available in RTD, we record the RTUC schedules for the second interval $(\mathrm{t}=2)$ and force them in the subsequent run. Therefore, we model $g e n_{g, t}, r s_{g, r e s, t}, u_{g, t}$ as parameters at $\mathrm{t}=1$ and as decision variables for $\mathrm{t}>1$.

A comparison of the aggregate cost under different uncertainty-related ramping requirements in absolute terms is informative; however, a comparison in relative (percentage) terms can be misleading because most aggregate cost is not caused by the uncertainty of net load or the procurement of uncertainty-related ramping products. That is why we propose and employ a metric called "uncertainty-induced costs." The "uncertainty-induced costs" are equal to (a) the aggregate cost of a simulation with net load uncertainty and ramping product minus (b) the aggregate cost of another simulation without uncertainty (i.e., perfect net load forecast). By subtracting term (b), this metric omits the portion of the aggregate cost that would be incurred if net load were known with certainty.

\section{NUMERICAL ILLUSTRATION OF VALUE OF RAMPING REQUIREMENT ESTIMATION METHODS}

We numerically illustrate the value of ramping sizing methods using a modified IEEE 118-bus system that mimics the annual generation mix of CAISO [19] with approximately $10 \%$ solar penetration (measured in terms of annual energy) in line with 2017 CAISO levels [20]. The case study closely follows the CAISO market structure in FESTIV [18] by simulating three markets: day-ahead with hourly resolution and a 24-hour horizon, RTUC with 15-min resolution and a 3hour horizon, and RTD with 5-min resolution and a 1-hour horizon. Moreover, to reflect the balancing nature of RTUC and RTD, we include CAISO must-run rules [3].

ISOs have multiple balancing products to address uncertainty and variability. Here, we focus on a single uncertainty - forecast errors of solar generation with a lead time of 2-3 hours - because novel methods aim to quantify uncertainty of solar irradiance [21]. In practice, ISOs account for uncertainty induced by gross load and wind forecast errors as well. We also estimate requirements for one product: the flexible ramping product (FRP) in RTUC. The 1-4 hour lead time of RTUC in our simulation ( $1-5$ hours in CAISO) facilitates the integration of weather-informed probabilistic forecasts with a lead time on the order of a few hours.

We assume that net load can take one of three values in each market interval $t$ (i.e., lower, mean, upper). We estimate RTUC FRP requirements in the down and up directions that address both forecasted movement from $t$ to $t+1$ and uncertainty at $t+1$ using (7) and (8) as follows. We set regulation and spinning reserves at $1 \%$ and $3 \%$ of gross load, respectively, and the value of lost load at $\$ 6,500 / \mathrm{MWh}$, which is the administrative penalty for power balance violations in the scheduling runs of CAISO [3]. We do not procure flexible ramping product in the RTD market because we assume that solar generation is known with certainty in the RTD market. 


$$
\begin{aligned}
& F R D R_{t}=\max \left(0, N L_{t, \text { mean }}-N L_{t+1, \text { lower }}\right) \\
& F R U R_{t}=\max \left(0, N L_{t+1, \text { upper }}-N L_{t, \text { mean }}\right)
\end{aligned}
$$

\section{A. Methods for Ramping Requirements Estimations}

To yield insights relevant for ongoing research, we estimate ramping requirements following two methods. The first method, hereafter called "alternative," incorporates probabilistic forecasting research results [22] by using forecasted percentiles of solar generation to estimate $N L_{t+1, \text { upper }}$ and $N L_{t+1, \text { lower }}(9)$. The second method, hereafter referred to as the "baseline", mimics industry practice and uses a histogram of past errors to estimate $N L_{t+1, \text { upper }}$ and $N L_{t+1, \text { lower }}(10)$ :

$$
\begin{aligned}
N L_{t+1, \text { upper } / \text { lower }}= & L O A D_{t+1}-W I N D_{t+1}-S O L A R_{t+1, Q_{5 / 95}} \\
N L_{t+1, \text { upper/lower }}= & L O A D_{t+1}-W_{t N D_{t+1}}-S O L A R_{t+1, \text { mean }} \\
& +E_{R R O R_{\text {up } / \text { down }}}
\end{aligned}
$$

This case study does not aim to propose or validate a comprehensive estimation method for ramping requirements; instead, it aims to demonstrate and discuss how system performance could change under an improved alternative method for the estimation of ramping requirements. Hence, to illustrate the procedure, we assume that the alternative method is perfectly accurate and by default better than the baseline method.

The baseline method collects net load forecast errors during a rolling window of 30 days for the same hour of the day and uses the third largest upward and downward errors ( $E R R O R_{\text {up } / \text { down }}$ ) to estimate the upper and lower value of net load, respectively. Here, we do not use realized values for past errors because sample errors would be large as we are considering only 4 days; instead, we assume that past errors follow a three-value discrete distribution with $5^{\text {th }}$ percentile, $95^{\text {th }}$ percentile, and mean equal to the ones estimated by the alternative method, and we construct a cumulative distribution function (CDF) for the third largest upward and downward past net load forecast error ${ }^{3}$ and select the median of that CDF as a proxy for the error in question.

This experimental design captures the essential differences between status quo and novel weather-informed methods. For instance, Fig. 2 contrasts requirements estimated by the baseline and alternative methods for 2 consecutive days (May 18 and 19, 2019). The 2 days have different weather conditions, as indicated by the mean forecast for photovoltaic (PV) generation (Fig. 2, top). The baseline method, however, estimates almost identical downward uncertainty for solar generation on both days (Fig. 2, middle) because it does not consider any information other than the hour of the day for grouping past errors. In contrast, the alternative method that uses the latest weather information (probabilistic solar power forecasts) estimates higher uncertainty during most hours of

\footnotetext{
${ }^{3}$ Under the three-value discrete distribution, the third largest error has $95 \%$ probability of estimating a positive error.

${ }^{4}$ The patterns of uncertainty realization are based on data from CAISO OASIS [24]. In detail, we estimate the error between the CAISO 15-min. real-time predispatch and 5-min RTD net load forecasts and compare it to $90 \%$ of the upward and downward uncertainty CAISO estimated for that interval. When
}

the day (especially during the middle of the day) on the day with a lower mean forecast of solar generation (Fig. 2, lower).
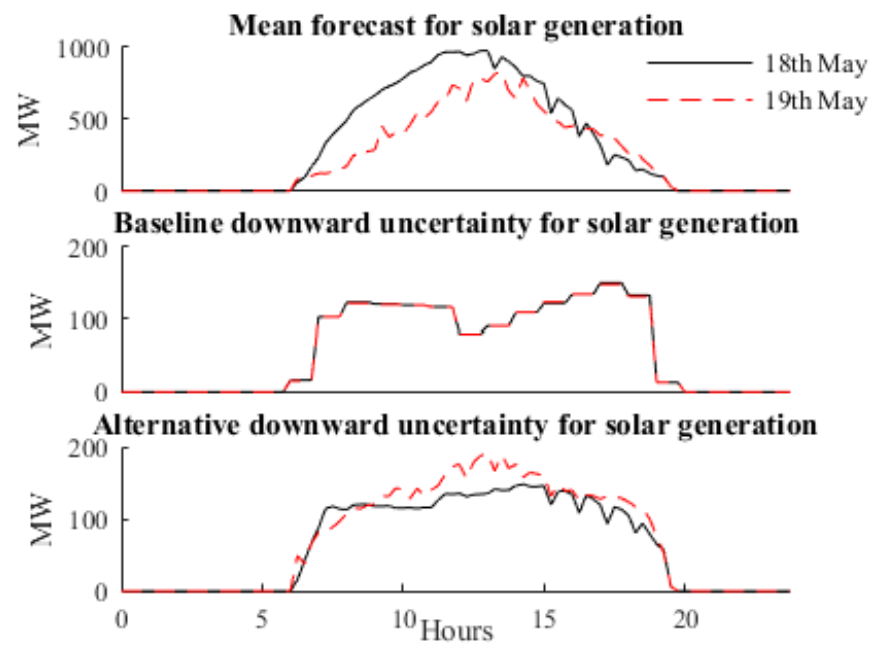

Figure 2. (top) Mean forecast for PV generation of test system on May 18 and 19; downward uncertainty for test system PV generation estimated by the baseline method (middle) and the alternative method (lower)

In situations similar to the one shown in Fig. 2, if the alternative method correctly estimates higher uncertainty on May 19, system reliability might be at risk when the baseline method is followed. Realized forecast errors are therefore necessary to assess system performance. Here, we develop a heuristic to create synthetic data for preliminary testing, but in future studies we will use realized values. In detail, we assume that the actual solar capacity is equal to either the lower $\left(S O L A R_{t, Q_{5}}\right)$, mean $\left(S O L A R_{t, \text { mean }}\right)$, or upper value $\left(S O L A R_{t, Q_{95}}\right)$ provided by the probabilistic forecasting method, and we choose one of those three values mimicking real patterns of uncertainty realization. ${ }^{4}$

We choose four simulation days that have different profiles, i.e., frequency of different type of intervals according to Fig. 1 (see Table I). On May 4 and 5, the alternative method estimates lower requirements than the baseline for most of the time (type: (b)). On May 19 and 20, the alternative method estimates higher requirements than the baseline for most of the daylight hours (type: (a)).

Table I. Profiles (Fig. 1) (\% of RTUC intervals in a1, a2, b1, b2, c)

\begin{tabular}{|c|c|c|c|c|c|}
\hline Day (May 2019) & a1 & a2 & b1 & b2 & c \\
\hline 4 & 1 & 7 & 13 & 36 & 43 \\
\hline 5 & 2 & 9 & 13 & 32 & 44 \\
\hline 19 & 14 & 21 & 3 & 19 & 44 \\
\hline 20 & 2 & 33 & 1 & 22 & 42 \\
\hline
\end{tabular}

\section{B. System Performance}

On May 4 and 5 when the alternative requirements are lower most of the time, we expect the alternative approach that uses weather-informed ramping requirements to yield

positive errors from real data exceed $90 \%$ of the upward uncertainty, we assume that the lower value of solar capacity is realized. When negative errors exceed $90 \%$ of the downward uncertainty, we assume that the upper value of solar capacity is realized. In all other cases, we assume that the mean value of solar capacity is realized. 
economic benefits because of lower FRP requirements. Results in Table II confirm our hypothesis for May 5 but contradict our hypothesis for May 4. Similarly, on May 19 and 20 when the alternative requirements are higher most of the time, we expect the alternative approach to yield reliability benefits that will partially or fully compensate for increased production costs. Results in Table II confirm our hypothesis for full compensation on May 19 and partial compensation on May 20. Note that the uncertainty-induced costs are a small portion of the total production cost $(<2 \%)$ at present renewable penetration levels, but the potential improvement or deterioration is significant.

Table II. Results per simulation day; deltas $(\Delta)$ are defined as the difference of (a) metric under the alternative simulation minus (b) metric under the baseline simulation.

\begin{tabular}{|c|c|c|c|c|}
\hline Day & $\begin{array}{c}\Delta \text { Production } \\
\text { Cost(\$) }\end{array}$ & $\begin{array}{c}\Delta \text { Unserved } \\
\text { Energy } \\
\text { (MWh) }\end{array}$ & $\begin{array}{c}\text { Baseline } \\
\text { uncertainty- } \\
\text { induced costs } \\
\text { (\% of baseline } \\
\text { aggregate cost) }\end{array}$ & $\begin{array}{c}\Delta \text { Uncertainty- } \\
\text { induced costs } \\
\text { (\% of baseline } \\
\text { uncertainty- } \\
\text { induced costs) }\end{array}$ \\
\hline 4 & 7,633 & 0 & 0.7 & $+150 \%$ \\
\hline 5 & $-6,702$ & 0 & 1.1 & $-83 \%$ \\
\hline 19 & 3,943 & -1.3 & 1.1 & $-55 \%$ \\
\hline 20 & 498 & 0 & 0.8 & $8 \%$ \\
\hline
\end{tabular}

Given that our hypothesis for anticipated performance was based on the most frequent type of interval, we further analyze the differences in production cost per type of interval, as shown in Table III. This detailed analysis helps explain the increase in production costs on May 4. In detail, production costs decrease as expected during intervals classified as type (b) and increase as expected during intervals classified as type (a); however, the increase in costs during the 8 intervals classified as type (a) exceeds the decrease in costs during the 47 intervals classified as type (b). This suggests that the procurement cost for flexibility is not uniform across hours and depends on system conditions. More than $50 \%$ of the cost increase under the alternative method on May 4 is caused by the nonconvex (startup and min. gen.) costs of a fast generator that is turned on to provide upward flexibility at 6 p.m.

Table III. Delta in production costs for 4 simulation days in May 2019 per type of interval (Fig.1)

\begin{tabular}{|c|c|c|c|c|c|}
\hline Day in May 2019 & a1 & a2 & b1 & b2 & c \\
\hline 4 & 5 & 28,329 & $-3,674$ & $-20,370$ & 3,344 \\
\hline 5 & -276 & $-18,208$ & 6,289 & 7,103 & $-1,609$ \\
\hline 19 & 548 & -649 & $-2,076$ & 6,079 & 41 \\
\hline 20 & 304 & $-1,818$ & 0 & 3,266 & $-1,254$ \\
\hline
\end{tabular}

The performance per type of interval (Table III) sometimes contradicts the hypotheses presented in Fig. 1. In detail, higher costs (positive deltas) are expected for (a1), but on May 5 lower costs are recorded. Similarly, higher costs are expected for (a2), but on May 5, 19, and 20 lower costs are recorded. In contrast, lower costs are expected under type (b), but higher costs are recorded on May 5, 19, and 20. At least two reasons explain this counterintuitive performance. First, RTUC optimizes costs over a multimarket interval horizon and intertemporal effects are possible. Increases in production cost during intervals of type (b2) might be caused by the presence of intervals of different type (here a2) in the same RTUC run. Second, the nonconvexity of commitment costs (startups and min-run) renders the allocation of costs to a specific interval difficult.
As shown in Table II, reliability performance improves, i.e., unserved energy reduces under the alternative set of requirements on May 19. The improvement in reliability is recorded for an interval of type (a1), which is consistent with our theoretical framework in Fig. 1; however, note that are additional intervals classified as type (a1) (Table I) during which reliability did not improve. At least two reasons explain this counterintuitive performance. First, the constraint for upward ramping procurement is not binding in any of those intervals in the baseline, and during most intervals the system has enough flexibility surplus that the realized ramping need (higher than baseline requirements) can be met. Second, the realized ramping need is sometimes lower than the alternative requirements because the system has already ramped up at a higher net load prior to that interval.

Last, even for the interval during which we observed the reliability improvement on May 19, the RTUC flexibility available under the baseline seems adequate to cover the realized ramping need; however, subsequent shutdowns make this flexibility nondeliverable. In our future work, we will investigate in detail causes of nondeliverability, such as transmission congestion and generation shutdowns; present side-by-side available RTUC flexibility and delivered RTD flexibility; and suggest alternative formulations for the procurement of deliverable ramping products.

In summary, more accurate estimation of ramping requirements could lead to production cost savings when requirements are lower (see type (b) intervals on May 4 and overall performance on May 5) or reliability improvements (see May 19) when requirements are higher; however, system conditions and formulations of ramping product affect the value of an alternative method.

\section{CONCLUSIONS}

We compared two alternative methods for the estimation of uncertainty-related ramping needs in terms of system performance theoretically and practically. For the theoretical comparison, we developed a framework that classifies market intervals into five types with different anticipated performance in terms of reliability and economics. We used the proposed framework to choose a few simulation days with different profiles (frequency of different types of intervals) for preliminary testing of new methods.

For the practical comparison, we focused on ramping requirements estimated a few hours in advance and contrasted two estimation methods: (a) a baseline (industry-inspired) method that considers calendar information and past errors and (b) an alternative (research-inspired) method that uses probabilistic weather-informed solar forecasts. Preliminary results on a 118-bus system suggest that weather-informed estimation methods could yield different ramping requirements than existing calendar-based methods. The changes in system performance varied significantly from one day to another. In a sample of 4 days in May 2019, the alternative method led to up to $83 \%$ lower and $150 \%$ higher uncertainty-induced costs than the baseline method. Moreover, preliminary results indicate that system conditions and ramping product design play a major role on the quantification of benefits. 
Preliminary results are encouraging for the development of more accurate ramping requirement estimation methods (possibly weather-informed) because improvement is expected during some days; however, simulations during a longer period (e.g., a year) are necessary. If net benefits are recorded under an improved method during a longer period, the quantification of the value will be useful to operators. If net costs are recorded under an improved method during a longer period, simulation results will be useful to (a) analysts for the identification of issues such as nondeliverability issues that prevent the realization of benefits and (b) forecasters for the identification of periods during which more accurate quantification could be valuable. Given the importance of system effects, in future work we will conduct simulations using larger and more realistic systems under a wide range of scenarios, including but not limited to varying penetration levels of renewable resources. We will also analyze the correlation of RTD power balance violations with traditional reliability metrics such as control performance standards [23] because RTD power balance violations (reliability metric used here) encompass information on both economic (out-of-market corrections) and reliability performance.

Finally, there are at least three open research questions for the development of practical weather-informed estimation methods for ramping requirements. First, methods that provide probabilistic forecasts of solar irradiance are yet to be thoroughly validated during long periods using metrics such as P-P plots and Brier scores [22]. Second, uncertainty related to wind and gross load should also be considered. Third, multiple balancing products address uncertainty of net load. Our future work will assess the potential of weather-informed methods for additional balancing products including RTD ramping and regulation using the framework shown in Fig.1.

\section{ACKNOWLEDGMENTS}

The authors gratefully acknowledge the contributions of Rui Zhang and Hendrik Hamman from IBM as well as Binghui $\mathrm{Li}$ and Jie Zhang from the University of Texas at Dallas in an ongoing project on probabilistic forecasting of solar irradiance. We also thank Amber Motley, Rebecca Webb, and Clyde Loutan at CAISO for feedback on earlier versions of this work.

This work was authored in part by the National Renewable Energy Laboratory, operated by Alliance for Sustainable Energy, LLC, for the U.S. Department of Energy (DOE) under Contract No. DE-AC36-08GO28308. Funding provided by U.S. Department of Energy Office of Energy Efficiency and Renewable Energy Solar Energy Technologies Office under Contract No. DE-EE0008215. The views expressed in the article do not necessarily represent the views of the DOE or the U.S. Government. The U.S. Government retains and the publisher, by accepting the article for publication, acknowledges that the U.S. Government retains a nonexclusive, paid-up, irrevocable, worldwide license to publish or reproduce the published form of this work, or allow others to do so, for U.S. Government purposes.

\section{REFERENCES}

[1] E. Ela et al., "Electricity markets and renewables: A Survey of potential design changes and their consequences," IEEE Power Energy Mag., vol. 15 , no. 6 , pp. 70-82, 2017.
[2] Midcontinent ISO, "Energy and Operating Reserve Markets," MISO, BPM-002-r18 Sep-23-2017, 2017.

[3] CAISO, "Business Practice Manual for Market Operations Version 60." 2019.

[4] Southwest Power Pool Market Monitoring Unit, "State of the market 2018," 2019.

[5] E. Ela and M. O'Malley, "Scheduling and Pricing for Expected Ramp Capability in Real-Time Power Markets," IEEE Trans. Power Syst., vol. 31, no. 3, pp. 1681-1691, 2016.

[6] B. Wang and B. F. Hobbs, "Real-Time Markets for Flexiramp: A Stochastic Unit Commitment-Based Analysis," IEEE Trans. Power Syst., vol. 31, no. 2, pp. 846-860, 2016.

[7] A. Daraeepour, D. Patino-Echeverri, and A. J. Conejo, "Economic and environmental implications of different approaches to hedge against wind production uncertainty in two-settlement electricity markets: A PJM case study," Energy Econ., vol. 80, pp. 336-354, 2019.

[8] K. H. Abdul-Rahman, H. Alarian, M. Rothleder, P. Ristanovic, B. Vesovic, and B. Lu, "Enhanced system reliability using flexible ramp constraint in CAISO market," in IEEE Power and Energy Society General Meeting, 2012.

[9] C. Wu, G. Hug, and S. Kar, "Risk-Limiting Economic Dispatch for Electricity Markets with Flexible Ramping Products," IEEE Trans. Power Syst., vol. 31, no. 3, pp. 1990-2003, 2016.

[10] N. Navid and G. Rosenwald, "Ramp Capability Product Design for MISO Markets," MISO, DRAFT, 2013.

[11] California ISO, "Flexible Ramping Product Draft Final Technical Appendix," CAISO, 2016.

[12] J. Dobschinski et al., "Uncertainty Forecasting in a Nutshell: Prediction Models Designed to Prevent Significant Errors," IEEE Power Energy Mag., vol. 15, no. 6, pp. 40-49, 2017.

[13] M. Poage and D. Tretheway, "Day-Ahead Market Enhancements," CAISO, 2019. [Online]. Available: www.caiso.com/Documents/PresentationDayAheadMarketEnhancemen tsWorkingGroupMeeting81319.pdf.

[14] P. Etingov et al., "Balancing needs assessment using advanced probabilistic forecasts," in 2018 International Conference on PMAPS 2018 - Proceedings, 2018, pp. 1-6.

[15] K. De Vos, N. Stevens, O. Devolder, A. Papavasiliou, B. Hebb, and J. Matthys-Donnadieu, "Dynamic dimensioning approach for operating reserves: Proof of concept in Belgium," Energy Policy, vol. 124, no. June 2018, pp. 272-285, 2019.

[16] EPEX SPOT, "XBID: CROSS-BORDER INTRADAY MARKET PROJECT.” [Online]. Available: https://www.epexspot.com/en/marketcoupling/xbid_cross_border_intraday_market_project

[17] E. Hirst and B. Kirby, "Separating and measuring the regulation and load-following ancillary services," Util. Policy, vol. 8, no. 2, pp. 75-81, 1999.

[18] E. Ela, M. Milligan, and M. O’Malley, “A flexible power system operations simulation model for assessing wind integration," in IEEE Power and Energy Society General Meeting, 2011.

[19] B.-M. Hodge, C. Brancucci Martinez-Anido, Q. Wang, E. Chartan, A. Florita, and J. Kiviluoma, "The combined value of wind and solar power forecasting improvements and electricity storage," Appl. Energy, vol. 214, no. March 2018, pp. 1-15, 2018.

[20] Department of Market Monitoring CAISO, "2017 Annual Report on Market Issues \& Performance," 2018.

[21] JHU IBM NREL UTD CAISO MISO, "Coordinated Ramping Product and Regulation Reserve Procurements in CAISO and MISO Using Multi-Scale Probabilistic Solar Power Forecasts Introduction,” 2018. [Online]. Available: www.energy.gov/sites/prod/files/2018/10/f56/Solar-Forecasting-2Kickoff-JHU.pdf.

[22] B. F. Hobbs et al., "Coordinated Ramping Product and Regulation Reserve Procurements in CAISO and MISO using Multi-Scale Probabilistic Solar Power Forecasts (Pro2R),” 2019. [Online]. Available: www.energy.gov/sites/prod/files/2019/10/f67/9 SolarForecasting-2-Annual-Review_The-Johns-Hopkins-University.pdf.

[23] NERC, "Reliability Standards for the Bulk Electric Systems of North America," North American Electric Reliability Corporation, Feb. 29, 2016.

[24] "CAISO Open Access Same-time Information System (OASIS)." [Online]. Available: http://oasis.caiso.com/mrioasis/logon.do. 